\title{
Association between prophylactic hydration volume and risk of contrast-induced nephropathy after emergent percutaneous coronary intervention
}

\author{
Tongtao Cui ${ }^{1,2 *}$, Jianbin $\mathrm{Zhao}^{1,2 *}$, Weijie Bei ${ }^{1,2 *}$, Hualong $\mathrm{Li}^{1,2}$, Ning Tan ${ }^{1,2 *}$, \\ Dengxuan $\mathrm{Wu}^{1}$, Kun Wang ${ }^{1,2}$, Xiaosheng Guo ${ }^{1,2}$, Yuanhui $\mathrm{Liu}^{1,2}$, \\ Chongyang Duan ${ }^{3}$, Shiqun Chen ${ }^{1,4}$, Kaiyang Lin ${ }^{1}$, Yong Liu ${ }^{1,2}$
}

${ }^{1}$ Department of Cardiology, Guangdong Cardiovascular Institute, Guangdong Provincial Key Laboratory of Coronary Disease, Guangdong General Hospital, Guangdong Academy of Medical Sciences,

School of Medicine, South China University of Technology, Guangzhou, China

${ }^{2}$ Southern Medical University, Guangzhou, China

${ }^{3}$ State Key Laboratory of Organ Failure Research, National Clinical Research Center for Kidney Disease, Department of Biostatistics, School of Public Health, Southern Medical University, Guangzhou, China ${ }^{4}$ Department of Biostatistics, South China College of Cardiovascular Research, Guangdong Society of Interventional Cardiology, Guangzhou, China

\begin{abstract}
Background: Intravenous hydration during percutaneous coronary intervention (PCI) significantly reduces the risk of contrast-induced nephropathy (CIN), but there are no well-defined protocols regarding the optimal hydration volume (HV) required to prevent CIN following emergent PCI. Therefore, this study investigates the association between the intravenous HV and CIN after emergent PCI.

Methods: 711 patients were prospectively recruited who had underwent emergent $P C I$ with hydration at routine speed and the relationship was investigated between $H V$ or $H V$ to weight ratio $(H V / W)$ and the CIN risk, which was defined as $a \geq 25 \%$ or $\geq 0.5 \mathrm{mg} / \mathrm{dL}$ increase in serum creatinine levels from baseline within 48-72 $h$ of exposure to the contrast.

Results: The overall CIN incidence was 24.7\%. Patients in the higher HV quartiles had elevated CIN rates. Multivariate analysis showed that higher $H V / W$ ratios were not associated with a decreased risk (using the $H V$ ) of CIN, but they were associated with an increased risk (using the $H V / W$ ) of $C I N$ (Q4 vs. Q1: adjusted odds ratio 1.99; 95\% confidence interval 1.05-3.74; $p=0.034$ ). A higher $H V / W$ ratio was not significantly associated with a reduced risk of long-term death (all $p>0.05$ ).

Conclusions: The data suggests that a higher total HV is not associated with a decreased CIN risk or beneficial long-term prognoses, and that excessive HV may increase the risk of CIN after emergent PCI. (Cardiol J 2017; 24, 6: 660-670)

Key words: contrast-induced nephropathy, hydration, emergent percutaneous coronary intervention, prognoses
\end{abstract}

Address for correspondence: Yong Liu, MD and Ning Tan, MD, Department of Cardiology, Guangdong Cardiovascular Institute, Guangdong Provincial Key Laboratory of Coronary Disease, Guangdong General Hospital, Guangdong Academy of Medical Sciences, School of Medicine, South China University of Technology, Guangzhou, 510100, China, tel: +86 15920172292; fax: +86-20-83824369, e-mails: liuyongmd@126.com (YL), tanning100@126.com (NT)

Received: 11.10.2016 Accepted: 21.12.2016

*These authors contributed equally to this work. 


\section{Introduction}

Contrast-induced nephropathy (CIN), which is a common complication following percutaneous coronary intervention (PCI), is associated with in-hospital and long-term morbidity and mortality, especially in high-risk patients undergoing emergent PCI [1-3] and hydration is the most effective method used to prevent CIN [4, 5]. Adequate intravenous administration of normal saline significantly reduced CIN risk in patients who underwent primary PCI (adequate hydration vs. no hydration: $11 \%$ vs. $21 \%$ ) [6]. Furthermore, a higher hydration volume (HV) has been associated with a significant trend towards a lower incidence of CIN [7]. However, no benefit existed in patients who were undergoing primary PCI and were administered more aggressive hydration [8].

Therefore, no well-defined protocols have been established regarding the optimal total HV and hydration duration for CIN prevention following emergent PCI. Since the hydration rate has been based on body weight (W) $(1 \mathrm{~mL} / \mathrm{kg} / \mathrm{h})$ in previous studies [6-9], we investigated the associations between the $\mathrm{HV}$ adjusted according to $\mathrm{W}(\mathrm{HV} / \mathrm{W}, \mathrm{mL} / \mathrm{kg})$ and the risk of $\mathrm{CIN}$ after emergent PCI.

\section{Methods}

This prospective observational study reviewed all consecutive patients who were candidates for emergent PCI between January 2010 and December 2013, using the institutional protocol described in a previous study [9]. In accordance with the updated guidelines [10], the study's exclusion criteria were pregnancy, lactation, the intravascular administration of a contrast medium within 7 days before the procedure or 3 days post-operatively $(\mathrm{n}=129)$, not using low-osmolarity contrast agents $(n=150)$, not undergoing emergent PCI $(\mathrm{n}=2890)$, cardiovascular surgery or endovascular repair $(n=384)$, end-stage renal disease or renal replacement $(n=9)$, missing pre- or postoperative creatinine data $(n=188)$, the presence of malignancies $(\mathrm{n}=3)$, not using isotonic saline for hydration $(n=28)$, and missing weight data $(n=1)$. Finally, 711 patients with ST-elevation myocardial infarction (STEMI) or those with non-ST-elevation acute coronary syndrome (NSTE-ACS) who were very high risk and underwent emergent PCI were included in the analysis (Fig. 1) [11].

Trained nurses carefully monitored and recorded the follow-up events through office visits and telephone interviews at $1,6,12,24$, and 36 months after the emergent PCI. The mean follow-up duration was $2.38 \pm 3.52$ years (median 2.08 years; interquartile range [IQR] 1.65-3.58 years). The institution's ethics research committee approved the study, and all patients gave their written informed consent to participate in this study.

Emergent PCI was defined as primary PCI for patients with STEMI and immediate PCI, that is, $<2 \mathrm{~h}$ from hospital admission and analogous to STEMI management, for very high-risk patients with NSTE-ACS [11]. Emergent PCI was performed using standard guide catheters, guide wires, balloon catheters, and stents that were inserted via the femoral or radial approaches. The contrast medium dose administered was left to the discretion of the interventional cardiologist. All patients received non-ionic, low-osmolarity contrast agents, either Iopamiron or Ultravist, both of which contain $370 \mathrm{mg}$ iodine $/ \mathrm{mL}$. The medications and invasive treatments were administered according to the American College of Cardiology Foundation/ American Heart Association guidelines [12]. In accordance with the local institutional protocol [9], the serum creatinine concentrations were measured in all patients on their admission to hospital, and on days 1, 2, and 3 after the emergent PCI.

The creatinine clearance $(\mathrm{CrCl})$ was calculated by applying the Cockcroft-Gault formula to the serum creatinine concentrations [13]. All patients received continuous intravenous infusions of isotonic saline, either at a rate of $1 \mathrm{~mL} / \mathrm{kg} / \mathrm{h}$ or at a rate of $0.5 \mathrm{~mL} / \mathrm{kg} / \mathrm{h}$ for cases with left ventricular ejection fractions (LVEF) of $<40 \%$ or severe congestive heart failure, which were initiated at or immediately before the emergent $\mathrm{PCI}$ procedure and continued for $4-24 \mathrm{~h}$ after the procedure. The $\mathrm{HV} / \mathrm{W}$ ratios were calculated using the $\mathrm{HV}$ and $\mathrm{W}$.

The primary endpoint was $\mathrm{CIN}_{25}$, which was defined as an increase in the serum creatinine level by $\geq 25 \%$ or $\geq 0.5 \mathrm{mg} / \mathrm{dL}$ from baseline within $72 \mathrm{~h}$ of contrast exposure [14]. Other CIN definitions included $\mathrm{CIN}_{0.5}$, which was defined as an increase of $\geq 0.5 \mathrm{mg} / \mathrm{dL}$ in the serum creatinine level from baseline within $72 \mathrm{~h}$ of contrast exposure [15] and $\mathrm{CIN}_{0.3}$, which was defined as an increase of $\geq 50 \%$ or $\geq 0.3 \mathrm{mg} / \mathrm{dL}$ in the serum creatinine level from baseline within $48 \mathrm{~h}$ of contrast exposure [16]. Also followed up on and recorded were in-hospital clinical outcomes, which included renal replacement therapy, acute heart failure, re-infarctions, the use of intra-aortic balloon pumps (IABP), arrhythmias, strokes, bleeding, and death, and long-term major adverse clinical events (MACE), which included 


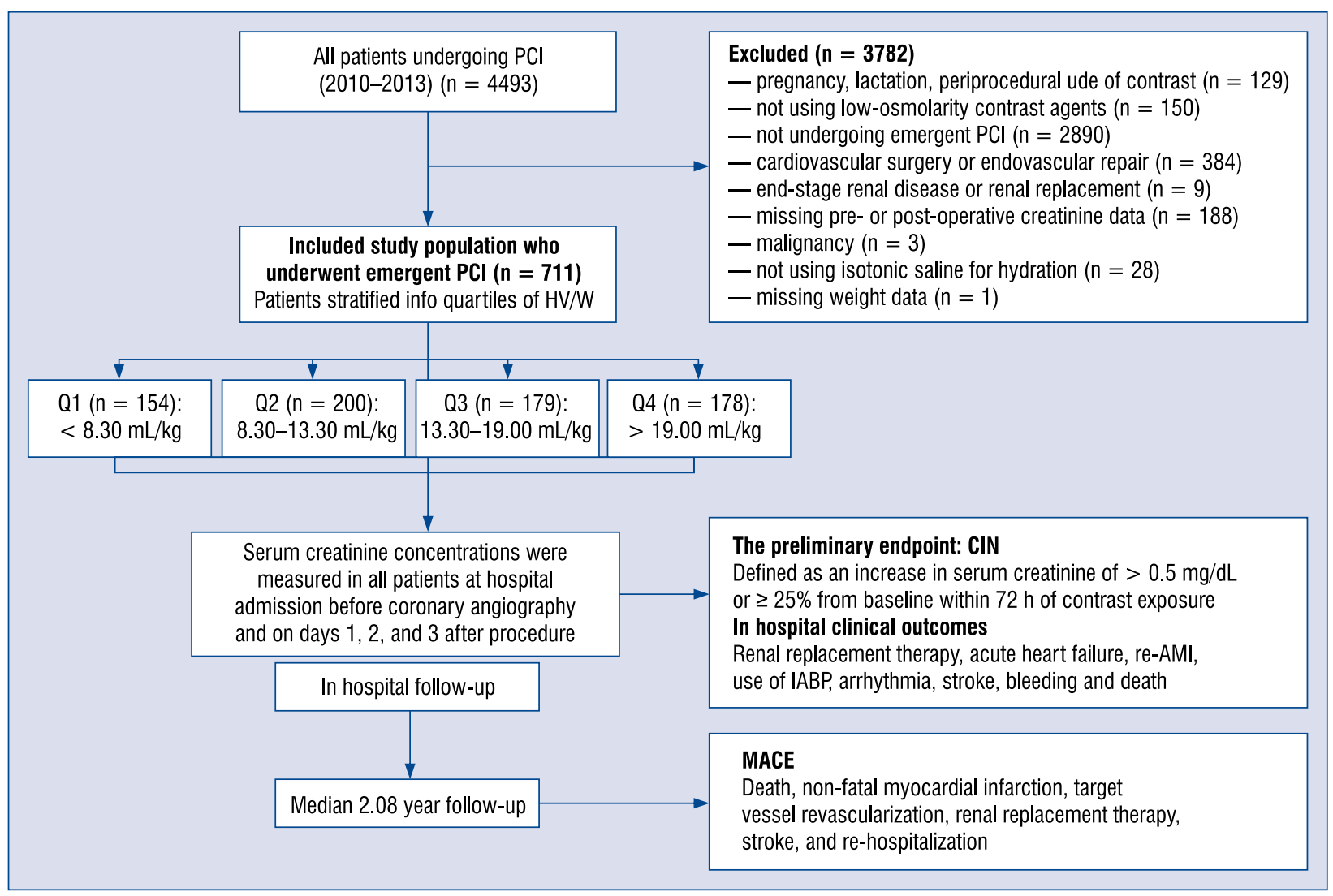

Figure 1. Flow chart of patients who underwent emergent percutaneous coronary intervention (PCI); $\mathrm{CIN}-$ contrast induced nephropathy; MACE - major adverse clinical events; HV/W - hydration volume to weight ratio; AMI acute myocardial infarction; IABP — intra-aortic balloon pump.

death, non-fatal myocardial infarction, target vessel revascularisation, $\mathrm{CIN}$ requiring renal replacement therapy, stroke, and re-hospitalisation.

\section{Statistical analysis}

For the continuous variables, one-way analyses of variance were conducted for the normally distributed data, which are expressed as the means \pm standard deviations, and the Kruskal-Wallis test was used to analyse the data with non-normal distributions, these are presented as medians and the IQR. Pearson's $\chi^{2}$ or Fisher's exact tests were used, as appropriate, to analyse the categorical variables, which are expressed as percentages. After trade-offs between over fitting and ensuring the unbalanced factors were well controlled, factors were used with $p$ values of $<0.05$ in the baseline analyses and the clinically important factors for multivariate logistic regression. Propensity score analysis was used for sensitivity analysis to compare CIN among groups. The Kaplan-Meier method and log-rank test were used to compare mortality and MACE rates according to the HV/W quartiles. In addition, multivariate Cox regression analyses, which were adjusted for age, a $\mathrm{CrCl}$ of $<60 \mathrm{~mL} / \mathrm{min}$, an LVEF $<40 \%$, anemia, diabetes mellitus, hypertension, and other factors, were conducted. The data were analysed on an available case basis, and patients with missing data were not included in the analysis. All of the data analyses were performed using SAS statistical software, version 9.4 (SAS Institute Inc., Cary, NC, USA) and $\mathrm{R}$ software, version 3.1.2 (The R Foundation for Statistical Computing Vienna, Austria; http:// www.R-project.org/) [17]. A two-sided p value $<0.05$ was considered statistically significant.

\section{Results}

\section{Baseline characteristics}

The patients $(\mathrm{n}=711)$ had a mean age of $62.21 \pm 12.30$ years, and there were 576 men who comprised $81 \%$ of the study population. $676(95.1 \%)$ patients were diagnosed with STEMI. All patients who underwent emergent PCI were stratified into the following quartiles based on their HV/W ratios: 
Table 1. Baseline patient characteristics according-the hydration volume-body weight ratio (HV/W) quartiles.

\begin{tabular}{|c|c|c|c|c|c|}
\hline \multirow[t]{2}{*}{ Characteristic } & \multicolumn{4}{|c|}{ HV/W quartiles } & \multirow{2}{*}{$\begin{array}{l}\text { P for } \\
\text { trend }\end{array}$} \\
\hline & $\begin{array}{c}\mathrm{Q1}(\mathrm{n}=154) \\
<8.30 \\
\mathrm{~mL} / \mathrm{kg}\end{array}$ & $\begin{array}{c}02(\mathrm{n}=200) \\
8.30-13.30 \\
\mathrm{~mL} / \mathrm{kg}\end{array}$ & $\begin{array}{c}\text { Q3 }(n=179) \\
13.30-19.00 \\
\mathrm{~mL} / \mathrm{kg}\end{array}$ & $\begin{array}{c}\mathrm{Q} 4(\mathrm{n}=178) \\
>19.00 \\
\mathrm{~mL} / \mathrm{kg}\end{array}$ & \\
\hline Sex, male & $133(86.4 \%)$ & $166(83.0 \%)$ & $140(78.2 \%)$ & $137(77.0 \%)$ & 0.103 \\
\hline Age [years] & $58.47 \pm 11.55$ & $62.44 \pm 11.37$ & $62.67 \pm 12.11$ & $64.79 \pm 13.39$ & $<0.001$ \\
\hline Age $>75$ years & $14(9.1 \%)$ & $30(15.0 \%)$ & $28(15.6 \%)$ & $45(25.3 \%)$ & $<0.001$ \\
\hline Weight [kg] & $70.33 \pm 9.71$ & $64.69 \pm 9.71$ & $64.07 \pm 11.10$ & $59.42 \pm 11.15$ & $<0.001$ \\
\hline STEMI & $144(94.1 \%)$ & $192(96.0 \%)$ & $168(93.9 \%)$ & $171(95.1 \%)$ & 0.66 \\
\hline Non-STEMI & $9(5.9 \%)$ & $8(4.0 \%)$ & $11(6.1 \%)$ & $7(4.9 \%)$ & 0.66 \\
\hline Systolic BP [mm Hg] & $125.51 \pm 20.69$ & $124.28 \pm 20.57$ & $121.50 \pm 22.51$ & $119.00 \pm 23.55$ & 0.028 \\
\hline LVEF [\%] & $55.25 \pm 10.17$ & $54.62 \pm 9.95$ & $54.19 \pm 11.24$ & $51.85 \pm 11.10$ & 0.025 \\
\hline LVEF $<40 \%$ & $9(6.5 \%)$ & $16(8.5 \%)$ & $23(13.9 \%)$ & $21(12.7 \%)$ & 0.113 \\
\hline $\mathrm{CHF}$ & $19(12.4 \%)$ & $51(25.5 \%)$ & $49(27.4 \%)$ & $52(29.2 \%)$ & 0.002 \\
\hline Post-IABP & $6(3.9 \%)$ & $17(8.5 \%)$ & $18(10.1 \%)$ & $27(15.2 \%)$ & 0.006 \\
\hline Mehran score & $4.28 \pm 3.75$ & $5.37 \pm 4.36$ & $5.89 \pm 5.10$ & $7.57 \pm 5.80$ & $<0.001$ \\
\hline \multicolumn{6}{|l|}{ Medical history: } \\
\hline Diabetes mellitus & $31(20.1 \%)$ & $47(23.5 \%)$ & $37(20.7 \%)$ & $34(19.1 \%)$ & 0.747 \\
\hline Smoker & $75(48.7 \%)$ & $89(44.5 \%)$ & $76(42.5 \%)$ & $82(46.1 \%)$ & 0.706 \\
\hline Hypertension & $64(41.6 \%)$ & $101(50.8 \%)$ & $91(50.8 \%)$ & $94(52.8 \%)$ & 0.179 \\
\hline Hypotension & $1(0.6 \%)$ & $11(5.5 \%)$ & $16(8.9 \%)$ & $27(15.2 \%)$ & $<0.001$ \\
\hline Hyperlipidemia & $24(15.6 \%)$ & $39(19.5 \%)$ & $35(19.6 \%)$ & $31(17.4 \%)$ & 0.746 \\
\hline Prior MI & $8(5.2 \%)$ & $10(5.0 \%)$ & $15(8.4 \%)$ & $6(3.4 \%)$ & 0.208 \\
\hline History of CABG & $0(0.0 \%)$ & $0(0.0 \%)$ & $1(0.6 \%)$ & $0(0.0 \%)$ & 0.395 \\
\hline \multicolumn{6}{|l|}{ Medication: } \\
\hline ACEI/ARB & $145(94.2 \%)$ & $184(92.0 \%)$ & $157(87.7 \%)$ & $141(79.2 \%)$ & $<0.001$ \\
\hline Beta-blocker & $136(88.3 \%)$ & $166(83.0 \%)$ & $136(76.0 \%)$ & $129(72.5 \%)$ & 0.001 \\
\hline CCB & $15(9.8 \%)$ & $26(13.1 \%)$ & $16(8.9 \%)$ & $13(7.3 \%)$ & 0.287 \\
\hline Diuretics & $40(26.0 \%)$ & $53(26.5 \%)$ & $84(46.9 \%)$ & $81(45.5 \%)$ & $<0.001$ \\
\hline \multicolumn{6}{|l|}{ Laboratory measurements: } \\
\hline Serum creatinine $[\mu \mathrm{mol} / \mathrm{L}]$ & $87.75 \pm 29.93$ & $92.63 \pm 35.18$ & $97.06 \pm 39.31$ & $106.43 \pm 53.02$ & $<0.001$ \\
\hline $\mathrm{CrCl}[\mathrm{mL} / \mathrm{min}]$ & $86.54 \pm 31.70$ & $71.67 \pm 26.91$ & $69.33 \pm 31.06$ & $59.74 \pm 30.57$ & $<0.001$ \\
\hline $\mathrm{CrCl}<60 \mathrm{~mL} / \mathrm{min}$ & $29(19.3 \%)$ & $71(35.9 \%)$ & $76(42.5 \%)$ & $99(56.6 \%)$ & $<0.001$ \\
\hline LDL-C & $3.21 \pm 1.11$ & $3.24 \pm 1.05$ & $3.27 \pm 1.02$ & $3.21 \pm 1.13$ & 0.976 \\
\hline HDL-C & $0.84 \pm 0.25$ & $0.97 \pm 0.24$ & $0.80 \pm 0.28$ & $0.92 \pm 0.36$ & 0.068 \\
\hline $\mathrm{TC}[\mathrm{mmol} / \mathrm{L}]$ & $4.93 \pm 1.24$ & $5.04 \pm 1.14$ & $4.83 \pm 1.10$ & $4.88 \pm 1.34$ & 0.688 \\
\hline $\mathrm{HbA} 1 \mathrm{c}[\%]$ & $6.67 \pm 1.58$ & $6.89 \pm 1.54$ & $6.48 \pm 1.44$ & $6.74 \pm 1.71$ & 0.237 \\
\hline hs-CRP [mg/L] & $16.48 \pm 24.62$ & $28.89 \pm 44.25$ & $30.71 \pm 35.45$ & $34.10 \pm 37.01$ & 0.077 \\
\hline Anemia & 45 (29.6\%) & $48(24.0 \%)$ & $48(26.8 \%)$ & $64(36.2 \%)$ & 0.063 \\
\hline Hematocrit [\%] & $41 \pm 5$ & $40 \pm 5$ & $40 \pm 5$ & $39 \pm 5$ & 0.031 \\
\hline
\end{tabular}

Data are presented as the means \pm standard deviations or as numbers and percentages. ACEl/ARB - angiotensin-converting enzyme inhibitor/angiotensin receptor blocker; $\mathrm{BP}$ - blood pressure; $\mathrm{CABG}$ — coronary artery bypass grafting; $\mathrm{CCB}$ - calcium channel blocker; $\mathrm{CHF}$ congestive heart failure; $\mathrm{CrCl}$ - creatinine clearance; $\mathrm{HbA1c}$ - glycated haemoglobin; $\mathrm{HDL}-\mathrm{C}$ - high-density lipoprotein cholesterol; hs-CRP — high sensitivity C-reactive protein; IABP — intra-aortic balloon pump, LDL-C — low-density lipoprotein cholesterol; LVEF — left ventricular ejection fraction; MI - myocardial infarction; STEMI - ST-segment elevation myocardial infarction; TC — total cholesterol

quartile $(\mathrm{Q}) 1:<8.30 \mathrm{~mL} / \mathrm{kg}$; Q2: 8.30-13.30 $\mathrm{mL} / \mathrm{kg}$; Q3: $13.30-19.00 \mathrm{~mL} / \mathrm{kg}$; and Q4: > $19.00 \mathrm{~mL} / \mathrm{kg}$. Patients with higher HV/W quartiles were significantly older, had worse renal functions, had lower
LVEF, higher contrast volumes and Mehran risk scores, and they used more IABP and diuretics, but fewer angiotensin-converting enzyme inhibitors and angiotensin receptor blockers (Tables 1,2). 
Table 2. Baseline procedural characteristics of hydration volume-body weight ratio (HV/W) quartiles (Q).

\begin{tabular}{|c|c|c|c|c|c|}
\hline \multirow[t]{2}{*}{ Characteristic } & \multicolumn{4}{|c|}{ HV/W quartiles } & \multirow{2}{*}{$\begin{array}{l}P \text { for } \\
\text { trend }\end{array}$} \\
\hline & $\begin{array}{c}\mathrm{Q1}(\mathrm{n}=154) \\
<8.30 \\
\mathrm{~mL} / \mathrm{kg}\end{array}$ & $\begin{array}{c}\mathrm{Q} 2(\mathrm{n}=200) \\
8.30-13.30 \\
\mathrm{~mL} / \mathrm{kg}\end{array}$ & $\begin{array}{c}\mathrm{Q} 3(\mathrm{n}=179) \\
13.30-19.00 \\
\mathrm{~mL} / \mathrm{kg}\end{array}$ & $\begin{array}{c}\mathrm{Q} 4(\mathrm{n}=178) \\
>19.00 \\
\mathrm{~mL} / \mathrm{kg}\end{array}$ & \\
\hline Diseased coronary lesion & $2.08 \pm 0.98$ & $2.07 \pm 1.31$ & $2.02 \pm 0.90$ & $2.22 \pm 0.99$ & 0.664 \\
\hline Number of stents & $1.15 \pm 0.72$ & $1.29 \pm 0.72$ & $1.37 \pm 0.83$ & $1.34 \pm 0.79$ & 0.060 \\
\hline Total length of stent [mm] & $30.40 \pm 23.06$ & $32.80 \pm 18.24$ & $36.23 \pm 23.35$ & $35.34 \pm 22.95$ & 0.290 \\
\hline Procedure duration [min] & $79.42 \pm 42.27$ & $76.08 \pm 32.49$ & $84.08 \pm 51.22$ & $83.82 \pm 33.00$ & 0.163 \\
\hline Contrast volume $[\mathrm{mL}]$ & $120.45 \pm 46.56$ & $124.35 \pm 46.92$ & $128.99 \pm 48.34$ & $129.52 \pm 46.23$ & 0.251 \\
\hline Contrast volume $>200 \mathrm{~mL}$ & $11(7.1 \%)$ & $15(7.5 \%)$ & $17(9.5 \%)$ & $19(10.7 \%)$ & 0.605 \\
\hline Hydration volume $[\mathrm{mL}]$ & $425.45 \pm 149.79$ & $674.49 \pm 137.65$ & $1027.35 \pm 189.39$ & $1741.96 \pm 808.48$ & $<0.001$ \\
\hline $\mathrm{HV} / \mathrm{W}[\mathrm{mL} / \mathrm{kg}]$ & $6.04 \pm 2.03$ & $10.43 \pm 1.51$ & $16.08 \pm 1.66$ & $29.74 \pm 13.39$ & $<0.001$ \\
\hline
\end{tabular}

Data are presented as the means \pm standard deviations or as numbers and percentages

Table 3. Incidence of contrast-induced nephropathy and in-hospital clinical outcomes according to hydration volume-body weight ratio (HV/W) quartiles (Q).

\begin{tabular}{|c|c|c|c|c|c|}
\hline \multirow[t]{2}{*}{ Characteristic } & \multicolumn{4}{|c|}{ HV/W quartiles } & \multirow{2}{*}{$\begin{array}{l}P \text { for } \\
\text { trend }\end{array}$} \\
\hline & $\begin{array}{c}\mathrm{Q1}(\mathrm{n}=154) \\
<8.30 \\
\mathrm{~mL} / \mathrm{kg}\end{array}$ & $\begin{array}{c}\mathrm{Q} 2(\mathrm{n}=200) \\
8.30-13.30 \\
\mathrm{~mL} / \mathrm{kg}\end{array}$ & $\begin{array}{c}\text { Q3 }(n=179) \\
13.30-19.00 \\
\mathrm{~mL} / \mathrm{kg}\end{array}$ & $\begin{array}{c}\mathrm{Q} 4(\mathrm{n}=178) \\
>19.00 \\
\mathrm{~mL} / \mathrm{kg}\end{array}$ & \\
\hline $\begin{array}{l}\text { SCr increase } \geq 0.5 \mathrm{mg} / \mathrm{dL} \\
\text { within } 72 \mathrm{~h}\end{array}$ & $1(0.7 \%)$ & $14(7.1 \%)$ & $12(6.7 \%)$ & $28(16.1 \%)$ & $<0.001$ \\
\hline $\begin{array}{l}\mathrm{SCr} \text { increase } \geq 0.5 \mathrm{mg} / \mathrm{dL} \\
\text { or } \geq 25 \% \text { within } 72 \mathrm{~h}\end{array}$ & $28(18.8 \%)$ & $48(24.2 \%)$ & $40(22.3 \%)$ & $57(32.8 \%)$ & 0.024 \\
\hline $\begin{array}{l}\mathrm{SCr} \text { increase } \geq 0.3 \mathrm{mg} / \mathrm{dL} \\
\text { or } \geq 50 \% \text { within } 48 \mathrm{~h}\end{array}$ & $12(8.1 \%)$ & $25(13.1 \%)$ & $20(11.3 \%)$ & $43(24.7 \%)$ & $<0.001$ \\
\hline IABP & $6(3.9 \%)$ & $18(9.0 \%)$ & $19(10.6 \%)$ & $27(15.2 \%)$ & 0.007 \\
\hline Reinfarction & $0(0.0 \%)$ & $1(0.5 \%)$ & $2(1.1 \%)$ & $2(1.1 \%)$ & 0.554 \\
\hline Stroke & $1(0.7 \%)$ & $2(1.0 \%)$ & $4(2.2 \%)$ & $4(2.2 \%)$ & 0.509 \\
\hline Acute heart failure & $6(3.9 \%)$ & $12(6.0 \%)$ & $16(8.9 \%)$ & $23(12.9 \%)$ & 0.014 \\
\hline Bleeding & $0(0.0 \%)$ & $1(0.5 \%)$ & $3(1.7 \%)$ & $12(6.7 \%)$ & $<0.001$ \\
\hline Death & $1(0.7 \%)$ & $4(2.0 \%)$ & $9(5.1 \%)$ & $28(15.7 \%)$ & $<0.001$ \\
\hline Require RRT & $2(1.3 \%)$ & $2(1.0 \%)$ & $1(0.6 \%)$ & $15(8.4 \%)$ & $<0.001$ \\
\hline
\end{tabular}

Data are presented as numbers and percentages; IABP — intra-aortic balloon pump; RRT — renal replacement therapy; SCr — serum creatinine

Hydration volume to weight ratio for predicting contrast-induced nephropathy

Patients with higher HV had higher CIN rates $(22.3 \%, 23.2 \%, 20.8 \%$, and $31.9 \%$ in $\mathrm{Q} 1, \mathrm{Q} 2, \mathrm{Q} 3$, and $\mathrm{Q} 4$, respectively; $\mathrm{p}=0.063$ ), and patients with higher $\mathrm{HV} / \mathrm{W}$ ratios had significantly higher CIN rates $(18.8 \%, 24.2 \%, 22.3 \%$, and $32.8 \%$ in Q1, Q2, $\mathrm{Q} 3$, and $\mathrm{Q} 4$, respectively; $\mathrm{p}=0.024$ ). Furthermore, the relationships between the HV or HV/W and CIN were consistent for different definitions of CIN, namely, $\mathrm{CIN}_{0.5}$ and $\mathrm{CIN}_{0.3}$ (Table 3).

Multivariate logistic regression analysis determined that higher $\mathrm{HV}$ were not associated with a reduced risk (using the $\mathrm{HV}$ ) of $\mathrm{CIN}_{25}$ (Q2 vs. Q1: odds ratio [OR] 1.06; 95\% confidence interval [CI] $0.60-1.85 ; \mathrm{p}=0.850 ; \mathrm{Q} 3$ vs. Q1: OR $0.68 ; 95 \% \mathrm{CI}$ $0.38-1.23 ; \mathrm{p}=0.203 ; \mathrm{Q} 4$ vs. Q1: OR $1.32 ; 95 \% \mathrm{CI}$ $0.77-2.26 ; \mathrm{p}=0.308$ ) (Table 4 ), but they were associated with an increased risk (using the HV/W) of $\mathrm{CIN}_{25}$ (Q4 vs. Q1: OR 1.99; 95\% CI 1.05-3.47; $\mathrm{p}=0.034$ ) (Table 4 ). Being female, aged $>75$ years, the $\mathrm{CrCl}$, and IABP and diuretic use were independent predictors of $\mathrm{CIN}_{25}$ (Table 5).

\section{In-hospital clinical outcomes}

In-hospital MACE occurred in 142 patients (20.0\%). Forty-two (5.9\%) patients died and 20 (2.8\%) patients required dialysis. Patients with 
Table 4. Univariate analyses and multivariate associations between contrast-induced nephropathy ${ }_{25}$ and the hydration volume (HV) to body weight (W) ratio quartiles (Q).

\begin{tabular}{|c|c|c|c|c|c|c|}
\hline \multirow[t]{2}{*}{ Risk factors } & \multicolumn{3}{|c|}{ Univariate logistic regression } & \multicolumn{3}{|c|}{ Multivariate logistic regression } \\
\hline & OR & $95 \% \mathrm{Cl}$ & $\mathbf{P}$ & OR & $95 \% \mathrm{Cl}$ & $\mathbf{P}$ \\
\hline HV/W ratio Q2 vs. Q1 & 1.38 & $0.82-2.34$ & 0.225 & 1.63 & $0.89-2.99$ & 0.113 \\
\hline HV/W ratio Q3 vs. Q1 & 1.24 & $0.72-2.14$ & 0.430 & 1.15 & $0.61-2.17$ & 0.665 \\
\hline HV/W ratio Q4 vs. Q1 & 2.11 & $1.25-3.54$ & 0.005 & 1.99 & $1.05-3.74$ & 0.034 \\
\hline Woman & 2.27 & $1.52-3.40$ & $<0.001$ & 2.84 & $1.72-4.71$ & $<0.001$ \\
\hline Age $>75$ years & 2.13 & $1.39-3.24$ & $<0.001$ & 2.49 & $1.44-4.31$ & 0.001 \\
\hline $\mathrm{CrCl}$ & 1.00 & $1.00-1.01$ & 0.645 & 1.02 & $1.01-1.02$ & $<0.001$ \\
\hline Anemia & 1.15 & $0.79-1.68$ & 0.451 & 1.09 & $0.69-1.71$ & 0.716 \\
\hline Diabetes mellitus & 1.02 & $0.67-1.55$ & 0.919 & 0.96 & $0.60-1.55$ & 0.871 \\
\hline Smoking & 0.88 & $0.62-1.24$ & 0.458 & 1.20 & $0.78-1.87$ & 0.408 \\
\hline Prior MI & 1.77 & $0.90-3.49$ & 0.098 & 1.57 & $0.72-3.43$ & 0.259 \\
\hline Hypotension & 2.01 & $1.13-3.56$ & 0.017 & 1.17 & $0.58-2.40$ & 0.658 \\
\hline LVEF & 0.97 & $0.95-0.99$ & 0.001 & 0.98 & $0.96-1.00$ & 0.058 \\
\hline Use of IABP & 3.57 & $2.16-5.92$ & $<0.001$ & 2.88 & $1.53-5.43$ & 0.001 \\
\hline Contrast volume & 1.00 & $1.00-1.01$ & 0.109 & 1.00 & $1.00-1.01$ & 0.184 \\
\hline Number of stents & 1.00 & $0.80-1.25$ & 0.982 & 0.95 & $0.74-1.24$ & 0.725 \\
\hline Use of diuretics & 1.78 & $1.25-2.52$ & 0.001 & 1.65 & $1.08-2.50$ & 0.019 \\
\hline Use of ACEl/ARB & 0.60 & $0.37-0.98$ & 0.043 & 0.82 & $0.44-1.52$ & 0.525 \\
\hline Use of $\beta$-blocker & 0.60 & $0.40-0.89$ & 0.011 & 0.81 & $0.49-1.33$ & 0.399 \\
\hline
\end{tabular}

$\mathrm{ACEI} / \mathrm{ARB}$ - angiotensin-converting enzyme inhibitor/angiotensin receptor blocker; $\mathrm{Cl}$ - confidence interval; $\mathrm{CrCl}$ - creatinine clearance; $\mathrm{HV} / \mathrm{W}$ - hydration volume to body weight ratio; IABP — intra-aortic balloon pump; LVEF — left ventricular ejection fraction; MI — myocardial infarction; OR - odds ratio

Table 5. Univariate analyses and multivariate associations between contrast-induced nephropathy ${ }_{25}$ and hydration volume (HV) ratio quartiles $(\mathrm{Q})$.

\begin{tabular}{|c|c|c|c|c|c|c|}
\hline \multirow[t]{2}{*}{ Risk factor } & \multicolumn{3}{|c|}{ Univariate logistic regression } & \multicolumn{3}{|c|}{ Multivariate logistic regression } \\
\hline & OR & $95 \% \mathrm{Cl}$ & $\mathbf{P}$ & OR & $95 \% \mathrm{Cl}$ & $\mathbf{P}$ \\
\hline HV ratio Q2 vs. Q1 & 1.06 & $0.64-1.75$ & 0.834 & 1.06 & $0.60-1.85$ & 0.850 \\
\hline HV ratio Q3 vs. Q1 & 0.92 & $0.55-1.52$ & 0.739 & 0.68 & $0.38-1.23$ & 0.203 \\
\hline HV ratio Q4 vs. Q1 & 1.63 & $1.03-2.58$ & 0.036 & 1.32 & $0.77-2.26$ & 0.308 \\
\hline Woman & 2.27 & $1.52-3.40$ & $<0.001$ & 3.00 & $1.81-4.97$ & $<0.001$ \\
\hline Age $>75$ years & 2.13 & $1.39-3.24$ & $<0.001$ & 2.47 & $1.43-4.29$ & 0.001 \\
\hline $\mathrm{CrCl}$ & 1.00 & $1.00-1.01$ & 0.645 & 1.01 & $1.01-1.02$ & $<0.001$ \\
\hline Anemia & 1.15 & $0.79-1.68$ & 0.451 & 1.04 & $0.66-1.62$ & 0.873 \\
\hline Diabetes mellitus & 1.02 & $0.67-1.55$ & 0.919 & 0.99 & $0.62-1.60$ & 0.973 \\
\hline Smoking & 0.88 & $0.62-1.24$ & 0.458 & 1.21 & $0.78-1.87$ & 0.392 \\
\hline Prior MI & 1.77 & $0.90-3.49$ & 0.098 & 1.51 & $0.69-3.31$ & 0.300 \\
\hline Hypotension & 2.01 & $1.13-3.56$ & 0.017 & 1.25 & $0.61-2.56$ & 0.544 \\
\hline LVEF & 0.97 & $0.95-0.99$ & 0.001 & 0.98 & $0.96-1.00$ & 0.053 \\
\hline Use of IABP & 3.57 & $2.16-5.92$ & $<0.001$ & 2.86 & $1.52-5.36$ & 0.001 \\
\hline Contrast volume & 1.00 & $1.00-1.01$ & 0.109 & 1.00 & $1.00-1.01$ & 0.171 \\
\hline Number of stents & 1.00 & $0.80-1.25$ & 0.982 & 0.97 & $0.75-1.26$ & 0.839 \\
\hline Use of diuretics & 1.78 & $1.25-2.52$ & 0.001 & 1.62 & $1.07-2.46$ & 0.023 \\
\hline Use of ACEl/ARB & 0.60 & $0.37-0.98$ & 0.043 & 0.83 & $0.44-1.55$ & 0.554 \\
\hline Use of $\beta$-blocker & 0.60 & $0.40-0.89$ & 0.011 & 0.77 & $0.47-1.27$ & 0.301 \\
\hline
\end{tabular}

$\mathrm{ACEl} / \mathrm{ARB}$ - angiotensin-converting enzyme inhibitors or angiotensin receptor blocker; $\mathrm{Cl}$ - confidence interval; $\mathrm{CrCl}-\mathrm{creatinine} \mathrm{clearance;}$ IABP -intra-aortic balloon pump; LVEF — left ventricular ejection fraction; MI - myocardial infarction; OR - odds ratio 


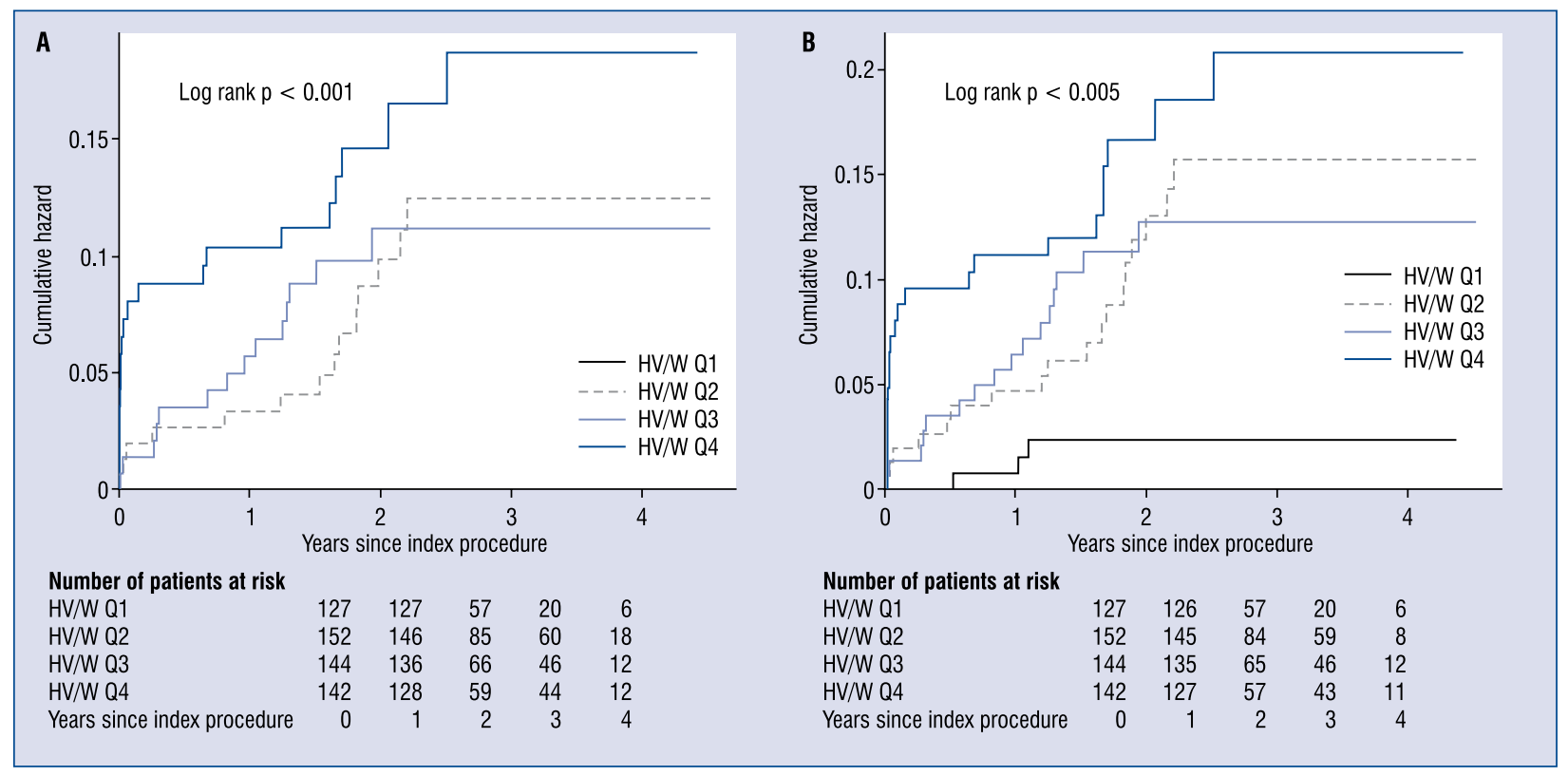

Figure 2. Kaplan-Meier curves for the cumulative probability of mortality and major adverse clinical events (MACE). A. Kaplan-Meier curves for the cumulative probability of mortality, B. Kaplan-Meier curves for the cumulative probability of MACE; HV/W - hydration volume to weight ratio.

elevated HV or HV/W ratios were more likely to experience MACE, which included acute heart failure (Q1: 3.9\%; Q2: 6.0\%; Q3: 8.9\%; and Q4: $12.9 \% ; \mathrm{p}=0.014)$, dialysis $(\mathrm{p}<0.001)$, and inhospital mortality (Q1: 0.7\%; Q2: 2.0\%; Q3: 5.1\%; and Q4: 15.7\%; $<$ 0.001) (Table 3).

\section{Follow-up clinical outcomes}

Median follow-up duration for this study cohort was 2.08 years (IQR $1.65-3.58$ years). Kaplan-Meier curve analyses revealed that higher HV/W ratios were significantly associated with increased risk of mortality $(\mathrm{p}<0.001)$ and MACE $(\mathrm{p}=0.005)$ (Fig. 2A, B). None of the patients in Q1 died. Cox proportional hazards modeling showed that after adjusting for confounding clinical factors that included hypertension, a $\mathrm{CrCl}<60 \mathrm{~mL} / \mathrm{min}$, use of diuetic bleeding, Mahran score and LVEF, the higher HV/W ratios were not associated with reduced risk of all-cause mortality (Q4 vs. Q2: adjusted hazard ratio $1.60 ; 95 \%$ CI 0.80 to 3.22 ; $\mathrm{p}=0.184)$ and MACE $(\mathrm{p}>0.05)$ (Fig. 3A, B).

Patients with $\mathrm{CIN}_{25}$ had increased risk of allcause mortality (Kaplan-Meier analysis $\mathrm{p}=0.001$; Cox regression analysis $\mathrm{p}=0.003$ ) and MACE (Kaplan-Meier analysis $\mathrm{p}=0.001$; Cox regression analysis $\mathrm{p}<0.001$ ) (Figs. 4, 5).

\section{Discussion}

The present study is the first to investigate the potential benefit of a higher HV in preventing CIN after emergent PCI when there is not enough time to administer pre-procedural hydration. It was found that even after adjusting for other risk confounders, patients with higher HV that had been adjusted according to their weight (HV/W ratios) did not appear to have lower risk for CIN or beneficial long-term prognoses. In addition, an excessive $\mathrm{HV}$ appeared to increase the risk of $\mathrm{CIN}$ after emergent PCI.

Patients undergoing emergent PCI procedures often have renal risk factors, including hypotension, shock, large volumes of contrast media administered, and the impossibility of starting renal prophylactic therapy [3] and they were also likely to present with reduced cardiac outputs and hypotension induced by myocardial infarction, and depletions in intravascular volume caused by vomiting, diaphoresis, or reduced oral intakes [7], which are associated with an increased risk of CIN $[3,7]$. The cumulative incidence of $\mathrm{CIN}$ was as high as $24.7 \%$ in the present study.

Intravenous hydration can inhibit the renin-angiotensin system and vasopressin, and it increased 


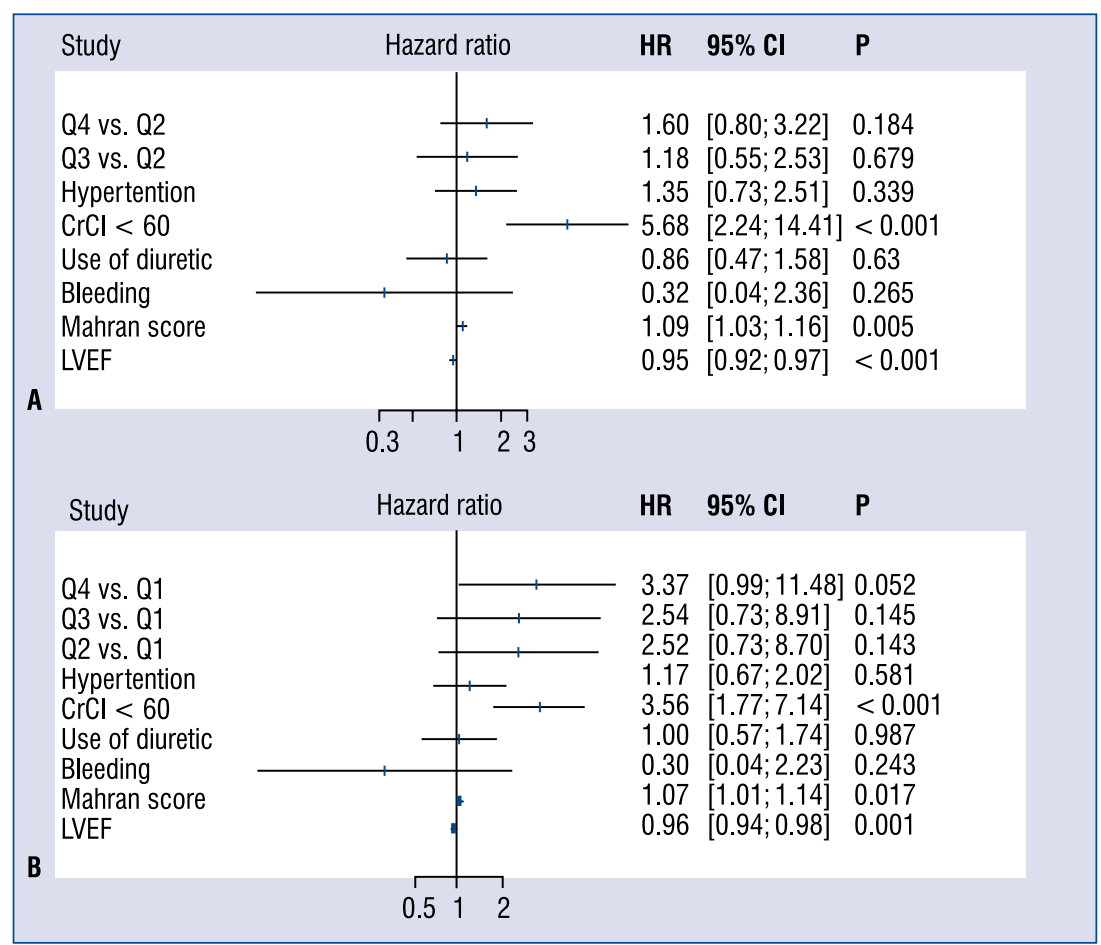

Figure 3. Multivariate Cox regression analysis indicated that higher hydration volume to body weight ratios were associated with increasing trends in all-cause mortality and major adverse clinical events (MACE). A. Multivariate Cox regression analysis for all-cause mortality, $\mathbf{B}$. Multivariate Cox regression analysis for $\mathrm{MACE} ; \mathrm{Cl}$ - confidence interval; $\mathrm{CrCl}$ - creatinine clearance; HR — hazard ratio; LVEF — left ventricular ejection fraction.

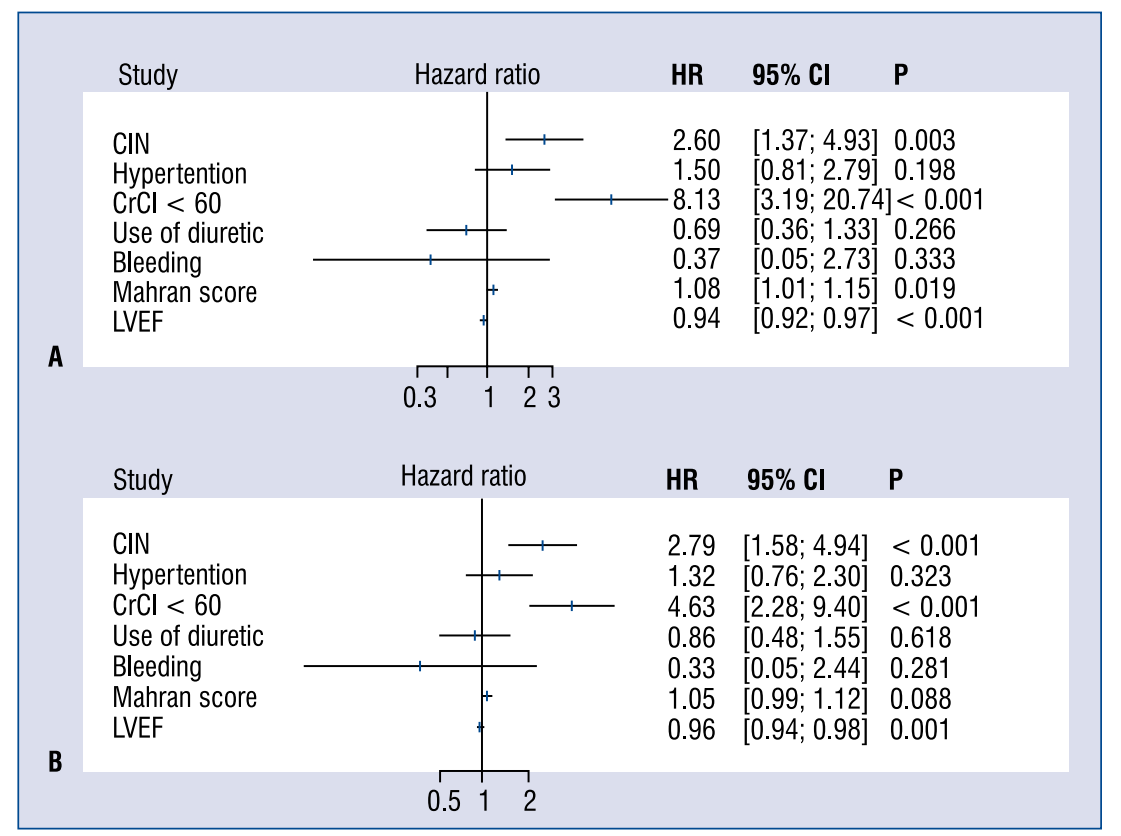

Figure 4. Multivariate Cox regression analysis indicated that higher hydration volume to body weight ratios were associated with increasing trends in all-cause mortality and major adverse clinical events (MACE). A. Multivariate Cox regression analysis for all-cause mortality, $\mathrm{B}$. Multivariate Cox regression analysis for $\mathrm{MACE}$; $\mathrm{Cl}$ - confidence interval; $\mathrm{CIN}$ - contrast induced nephropathy; HR — hazard ratio; LVEF — left ventricular ejection fraction. 

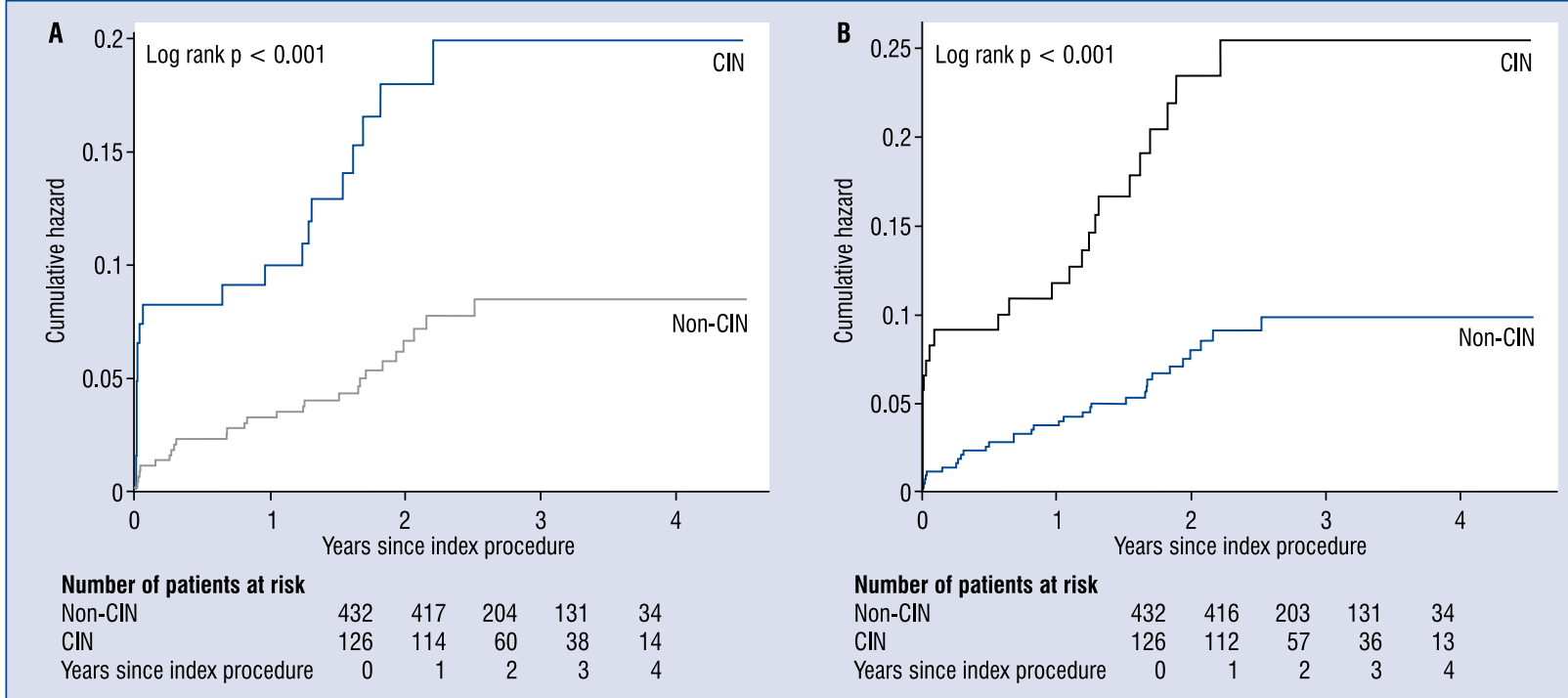

Figure 5. Kaplan-Meier curves for the cumulative probability of mortality and major adverse clinical events (MACE). A. Kaplan-Meier curves for the cumulative probability of mortality, B. Kaplan-Meier curves for the cumulative probability of MACE; CIN — contrast induced nephropathy.

the urinary flow rate, reduced the concentration of the contrast medium in the tubules, and it expedited the excretion of the contrast medium, thereby reducing the length of time that the tubular cells were exposed to the toxic effects of the contrast medium [18]. Maioli et al. [7] assigned patients to the following HV tertiles: $1^{\text {st }}$ tertile: $\leq 900 \mathrm{~mL}$; $2^{\text {nd }}$ tertile: $901-1080 \mathrm{~mL}$; and $3^{\text {rd }}$ tertile: $\geq 1081 \mathrm{~mL}$, and they demonstrated a significant trend between lower CIN incidence rates and increasing HV, with contrast-induced acute kidney injury (CI-AKI) rates of $26.7 \%, 17 \%$, and $8.1 \%$, respectively, and an $\mathrm{HV} \leq 960 \mathrm{~mL}$ was the optimal cut-off point for predicting the risk of CI-AKI. However, different agents, namely, saline and bicarbonate, were used for hydration, and normal saline is more readily available in emergent clinical practice, accordingly this study analysed the association between the saline HV and CIN, but consistent benefits of higher HV were not evident.

Negative results in the present study are consistent with those reported from studies on patients with chronic kidney disease in which $20 \mathrm{~h}$ of pre-procedural hydration with $1 \mathrm{~mL} / \mathrm{kg} / \mathrm{h}$ isotonic saline was similar to $5 \mathrm{~h}$ of pre-procedural hydration in relation to the prevention of CIN [19] and $24 \mathrm{~h}$ of hydration before plus $48 \mathrm{~h}$ of hydration after the procedure with $1 \mathrm{~mL} / \mathrm{kg} / \mathrm{h}$ isotonic saline (highdose group $5334 \pm 783 \mathrm{~mL}$ ) did not appear to provide additional benefits in terms of CIN prevention compared with $12 \mathrm{~h}$ of hydration before plus $12 \mathrm{~h}$ of hydration with $1 \mathrm{~mL} / \mathrm{kg} / \mathrm{h}$ isotonic saline (low-dose group $1893 \pm 270 \mathrm{~mL}$ ) after coronary procedures [20]. Given the short post-procedural half-lives of contrast media, short-term peri-procedural hydration rather than long-term hydration could be more effective at reducing the risk of CIN [21]. Following the short period of hydration required for the clearance of contrast medium, continuous long-term hydration may have no additional benefits in the prevention of CIN.

Findings of a recent study have also shown that a higher saline HV $(1085 \pm 168 \mathrm{~mL}$ vs. $941 \pm 148 \mathrm{~mL}$ ) did not reduce the rate of CI-AKI (19\% vs. $19.2 \%$ ) following primary angioplasty, even with rapid hydration before the procedure [8]. These findings are similar to those from the present study in which similar patients underwent emergent procedures. Experimental studies have established that contrast media induce dose-dependent and time-dependent renal cell injuries [22]. If renal cell toxicity begins a few minutes after the cells have been exposed to the contrast medium, it can be speculated that even high HV initiated simultaneously might be insufficient to counter the cytotoxic effects of the contrast medium.

In patients within the higher HV/W quartiles in the present study, the higher risk of CIN seemed to correlate strongly with the more harmful clinical characteristics that included older age, lower 
LVEF and $\mathrm{CrCl}$, a higher frequency of congestive heart failure, diabetes, emergent PCI, and higher contrast volumes. However, higher HV/W ratios seemed to be associated with an increasing trend of CIN incidence even after adjusting for the above confounding variables. Indeed, an excessive $\mathrm{HV}$ seems to increase the risks of CIN and poor clinical outcomes by mechanisms that are not yet fully understood. An excessive HV that is administered at a standard speed may increase the risk of heart failure, which may lead to overload volumes or increases in the preloads, lower cardiac outputs, and inadequate renal perfusion, as it occurs in cardiorenal syndrome, which, in turn, may increase the venous pressure, leading to kidney congestion, activation of the renin-angiotensin-aldosterone system, and marked alterations in immune and somatic cell signalling [23, 24]. Hence, it is likely that higher $\mathrm{HV}$ administered at average speeds did not have any benefits in relation to prevention of CIN or patient prognoses. In the present study however, the rates of hydration were reduced by half in cases with an LVEF of $<40 \%$ or in those with severe congestive heart failure. Moreover, the so-called force diuresis may be another factor of renal function deterioration after contrast medium exposure. As mentioned above, an excessive HV may lead to overload volumes. To avoid excessive positive fluid balance, diuretics were more common use in patients with higher $\mathrm{HV}$, as shown in the results of our study (from Q1 to Q4, 26.0 vs. 26.5 vs. 46.9 vs. $45.5 \%, \mathrm{p}<0.001$ ). Indeed, previous clinical studies demonstrated that the prophylactic furosemide appears to increase CIN rate in patients without adequate matching between hydration and urine flow [25, 26]. Drastically forced diuresis induced reduction of blood circulation volume and dehydration, which may deteriorate renal hemodynamics and activate renin-angiotensin system [27]. As a result, the contrast induced kidney was exacerbated.

Recently, peri-procedural, short-term, and rapid hydration with normal saline has demonstrated remarkable benefits with respect to reducing the CIN risk [28, 29]. Shorter peri-procedural hydration effectively reduces the viscosity of the urine, which, in turn, may accelerate the excretion of the contrast medium [30,31]. Hence, rather than administering fluid at an average rate over a long period of time, more rapid hydration may help to attenuate the increase in urine viscosity caused by the administration of contrast medium, thereby aiding contrast medium excretion and shortening the exposure time of the tubular cells to contrast medium. Further randomized studies are needed to clarify the relationship between the HV and CIN after emergent PCI.

\section{Limitations of the study}

The current study has several limitations. First, this prospective observational study was conducted at a single centre; therefore, baseline deviation existed inevitably (such as men comprising $81 \%$ of the study population) and the evidence may not be as robust as that obtained from a randomized, controlled trial. Second, $\mathrm{CrCl}$ was obtained using the Cockcroft-Gault formula rather than by undertaking direct measurements. Third, variations in the times at which the measurements were undertaken may have missed the post-procedural peak creatinine levels. Finally, data that described patient oral water intakes were absent; hence, the inability to investigate whether patients with low HV drank more water and achieved higher HV that were potentially similar to those administered higher intravenous HV.

\section{Conclusions}

In conclusion, the data suggests that higher $\mathrm{HV}$ achieved through the administration of normal saline at a standard speed and adjusted according to $\mathrm{W}$ showed no benefit in relation to the prevention of CIN or in relation to long-term prognosis after emergent PCI, but excessive hydration appeared to be associated with an increased risk of CIN in patients undergoing emergent PCI. Randomised studies are needed to clarify the relationship between the HV and CIN after emergent PCI.

\section{Acknowledgements}

This study was supported by grants from the Science and Technology Planning Project of Guangdong Province (Grant number: 2008A030201002) and the Guangdong Cardiovascular Institute and Guangdong Provincial Cardiovascular Clinical Medicine Research Fund (Grant number: 2009X41). The funders had no role in study design, data collection, analysis, decision to publish, or the preparation of the manuscript.

Preliminary work on this study was accepted and will be presented in abstract form at the ESC Congress 2016, European Society of Cardiology; August 27-31, 2016, Rome, Italy.

Conflict of interest: None declared 


\section{References}

1. Aurelio A, Durante A. Contrast-induced nephropathy in percutaneous coronary interventions: pathogenesis, risk factors, outcome, prevention and treatment. Cardiology. 2014; 128(1): 62-72, doi: 10.1159/000358042, indexed in Pubmed: 24557146.

2. McCullough PA. Contrast-induced acute kidney injury. J Am Coll Cardiol. 2008; 51(15): 1419-1428, doi: 10.1016/j.jacc.2007.12.035, indexed in Pubmed: 18402894.

3. Marenzi G, Lauri G, Assanelli E, et al. Contrast-induced nephropathy in patients undergoing primary angioplasty for acute myocardial infarction. J Am Coll Cardiol. 2004; 44(9): 1780-1785, doi: 10.1016/j. jacc.2004.07.043, indexed in Pubmed: 15519007.

4. O'Gara PT, Kushner FG, Ascheim DD, et al. 2013 ACCF/AHA guideline for the management of ST-elevation myocardial infarction: Executive summary. A report of the American College of Cardiology Foundation/American Heart Association Task Force on Practice Guidelines. J Am Coll Cardiol. 2013; 61(4): e78-e140, doi: 10.1016/j. jacc.2012.11.018.

5. Cecere N, Jadoul M, Labriola L. Intravenous hydration (with or without rosuvastatin) should remain the cornerstone of the prevention of contrast-induced acute kidney injury in patients with diabetes and chronic kidney disease. J Am Coll Cardiol. 2014; 64(3): 332, doi: 10.1016/j.jacc.2014.02.614, indexed in Pubmed: 25034074.

6. Jurado-Román A, Hernández-Hernández F, García-Tejada J, et al. Role of hydration in contrast-induced nephropathy in patients who underwent primary percutaneous coronary intervention. Am J Cardiol. 2015; 115(9): 1174-1178, doi: 10.1016/j.amjcard.2015.02.004, indexed in Pubmed: 25759106.

7. Maioli M, Toso A, Leoncini M, et al. Effects of hydration in contrast-induced acute kidney injury after primary angioplasty: a randomized, controlled trial. Circ Cardiovasc Interv. 2011; 4(5): 456-462, doi: 10.1161/CIRCINTERVENTIONS.111.961391, indexed in Pubmed: 21972403 .

8. Manari A, Magnavacchi P, Puggioni E, et al. Acute kidney injury after primary angioplasty: effect of different hydration treatments. J Cardiovasc Med (Hagerstown). 2014; 15(1): 60-67, doi: 10.2459/ JCM.0b013e3283641bb8, indexed in Pubmed: 24500238.

9. Liu Y, Chen JY, Tan N, et al. Safe limits of contrast vary with hydration volume for prevention of contrast-induced nephropathy after coronary angiography among patients with a relatively low risk of contrast-induced nephropathy. Circ Cardiovasc Interv. 2015; 8(6), doi: 10.1161/CIRCINTERVENTIONS.114.001859, indexed in Pubmed: 26041500 .

10. Stacul F, van der Molen AJ, Reimer P, et al. Contrast Media Safety Committee of European Society of Urogenital Radiology (ESUR). Contrast induced nephropathy: updated ESUR Contrast Media Safety Committee guidelines. Eur Radiol. 2011; 21(12): 2527-2541, doi: 10.1007/s00330-011-2225-0, indexed in Pubmed: 21866433.

11. Hamm CW, Bassand JP, Agewall S, et al. ESC Committee for Practice Guidelines. ESC Guidelines for the management of acute coronary syndromes in patients presenting without persistent ST-segment elevation: The Task Force for the management of acute coronary syndromes (ACS) in patients presenting without persistent ST-segment elevation of the European Society of Cardiology (ESC). Eur Heart J. 2011; 32(23): 2999-3054, doi: 10.1093/eurheartj/ehr236, indexed in Pubmed: 21873419.

12. Wright RS, Anderson JL, Adams CD, et al. 2011 ACCF/AHA focused update of the Guidelines for the Management of Patients with Unstable Angina/Non-ST-Elevation Myocardial Infarction (updating the 2007 guideline): a report of the American College of Cardiology Foundation/American Heart Association Task Force on Practice Guidelines developed in collaboration with the American College of Emergency Physicians, Society for Cardiovascular Angiography and Interventions, and Society of Thoracic Surgeons. J Am Coll Cardiol. 2011; 57(19): 1920-1959, doi: 10.1016/j.jacc.2011.02.009, indexed in Pubmed: 21450428.

13. Xun L, Cheng W, Hua T, et al. Assessing glomerular filtration rate (GFR) in elderly Chinese patients with chronic kidney disease (CKD): a comparison of various predictive equations. Arch Gerontol Geriatr. 2010; 51(1): 13-20, doi: 10.1016/j.archger.2009.06.005, indexed in Pubmed: 19615764.

14. Ivanes F, Isorni MA, Halimi JM, et al. Predictive factors of contrastinduced nephropathy in patients undergoing primary coronary angio- plasty. Arch Cardiovasc Dis. 2014; 107(8-9): 424-432, doi: 10.1016/j. acvd.2014.05.008, indexed in Pubmed: 25082735.

15. Barrett BJ, Parfrey PS. Clinical practice. Preventing nephropathy induced by contrast medium. N Engl J Med. 2006; 354(4): 379-386, doi: 10.1056/NEJMcp050801, indexed in Pubmed: 16436769.

16. Moriyama N, Ishihara M, Noguchi T, et al. Admission hyperglycemia is an independent predictor of acute kidney injury in patients with acute myocardial infarction. Circ J. 2014; 78(6): 1475-1480, indexed in Pubmed: 24694768.

17. R: A language and environment for statistical computing. R Foundation for Statistical Computing, Vienna, Austria. 2014. http://www.R-project.org/.

18. Weisbord SD, Palevsky PM. Prevention of contrast-induced nephropathy with volume expansion. Clin J Am Soc Nephrol. 2008; 3(1) 273-280, doi: 10.2215/CJN.02580607, indexed in Pubmed: 17989201.

19. Torigoe K, Tamura A, Watanabe T, et al. 20-Hour preprocedural hydration is not superior to 5-hour preprocedural hydration in the prevention of contrast-induced increases in serum creatinine and cystatin C. Int J Cardiol. 2013; 167(5): 2200-2203, doi: 10.1016/j. ijcard.2012.05.122, indexed in Pubmed: 22717305.

20. Koc F, Ozdemir K, Kaya MG, et al. Intravenous N-acetylcysteine plus high-dose hydration versus high-dose hydration and standard hydration for the prevention of contrast-induced nephropathy: CASIS - a multicenter prospective controlled trial. Int J Cardiol. 2012; 155(3): 418-423, doi: 10.1016/j.ijcard.2010.10.041, indexed in Pubmed: 21106264 .

21. Briguori C, Condorelli G. Hydration in contrast-induced acute kidney injury. Lancet. 2014; 383: 1786-1788.

22. Romano G, Briguori C, Quintavalle C, et al. Contrast agents and renal cell apoptosis. Eur Heart J. 2008; 29(20): 25692576, doi: 10.1093/eurheartj/ehn197, indexed in Pubmed: 18468994.

23. House AA, Anand I, Bellomo R, et al. Acute Dialysis Quality Initiative Consensus Group. Definition and classification of cardio-renal syndromes: workgroup statements from the 7th ADQI Consensus Conference. Nephrol Dial Transplant. 2010; 25(5): 1416-1420, doi: 10.1093/ ndt/gfq136, indexed in Pubmed: 20228069.

24. Ronco C, Cicoira M, McCullough PA. Cardiorenal syndrome type 1: Pathophysiological crosstalk leading to combined heart and kidney dysfunction in the setting of acutely decompensated heart failure. J Am Coll Cardiol. 2012; 60(12): 1031-1042, doi: 10.1016/j.jacc.2012.01.077, indexed in Pubmed: 22840531.

25. Dussol B, Morange S, Loundoun A, et al. A randomized trial of saline hydration to prevent contrast nephropathy in chronic renal failure patients. Nephrol Dial Transplant. 2006; 21(8): 2120-2126, doi: 10.1093/ ndt/gfl133, indexed in Pubmed: 16611682.

26. Solomon R, Werner C, Mann D, et al. Effects of saline, mannitol, and furosemide on acute decreases in renal function induced by radiocontrast agents. N Engl J Med. 1994; 331(21): 1416-1420, doi: 10.1056 NEJM199411243312104, indexed in Pubmed: 7969280.

27. Majumdar SR, Kjellstrand CM, Tymchak WJ, et al. Forced euvolemic diuresis with mannitol and furosemide for prevention of contrastinduced nephropathy in patients with CKD undergoing coronary angiography: a randomized controlled trial. Am J Kidney Dis. 2009; 54(4): 602-609, doi: 10.1053/j.ajkd.2009.03.024, indexed in Pubmed: 19535188 .

28. Briguori C, Visconti G, Focaccio A, et al. REMEDIAL II Investigators. Renal insufficiency after contrast media administration tria II (REMEDIAL II): RenalGuard System in high-risk patients for contrast-induced acute kidney injury. Circulation. 2011; 124(11): 1260-1269, doi: 10.1161/CIRCULATIONAHA.111.030759, indexed in Pubmed: 21844075.

29. Brar SS, Aharonian V, Mansukhani P, et al. Haemodynamic-guided fluid administration for the prevention of contrast-induced acute kidney injury: the POSEIDON randomised controlled trial. Lancet. 2014; 383(9931): 1814-1823, doi: 10.1016/S0140-6736(14)60689-9, indexed in Pubmed: 24856027.

30. Seeliger E, Becker K, Ladwig M, et al. Up to 50-fold increase in urine viscosity with iso-osmolar contrast media in the rat. Radiology. 2010; 256(2): 406-414, doi: 10.1148/radiol.10091485, indexed in Pubmed: 20529990.

31. Seeliger E, Sendeski M, Rihal CS, et al. Contrast-induced kidney injury: mechanisms, risk factors, and prevention. Eur Heart J. 2012 33(16): 2007-2015, doi: 10.1093/eurheartj/ehr494, indexed in Pubmed: 22267241 . 